\title{
DETERMINAÇÃO DA DEMANDA QUÍMICA DE OXIGÊNIO (DQO) EM EFLUENTES A PARTIR DA AQUISIÇÃO DE IMAGENS DIGITAIS UTILIZANDO SMARTPHONE
}

\author{
RADTKE, J. F.1; MARTINS, J. S. ${ }^{2}$. MACHADO, E. L. ${ }^{2}$. \\ PALAVRAS CHAVE: Imagem digital. Demanda Química de Oxigênio (DQO). Efluente.
}

\begin{abstract}
RESUMO
A Demanda Química de Oxigênio (DQO) é um indicador global de matéria orgânica presente em águas residuais e superficiais, sendo amplamente utilizada em efluentes líquidos que monitoram a descarga de efluentes líquidos. 0 objetivo deste trabalho foi desenvolver um método alternativo de espectrofotometria para leitura e determinação de DQO em efluentes urbanos, utilizando aquisição de imagens digitais por smartphone e o tratamento das mesmas por meio de ImageJ e Photometrix. Através da construção de curvas analíticas foi possível validar o método utilizando o vetor Photometrix e RGB, resultando em um intervalo de trabalho linear de $55-600 \mathrm{mg} \mathrm{L}^{-1}$ de $\mathrm{O}_{2}$ e um método mais sensível, preciso e exato do que o método padrão referenciado. 0 uso de amostras reais também obteve melhores resultados com este método, com recuperação média de $93,5 \%(n=4)$, capaz de quantificar $o$ analito em amostras de efluentes com a mesma confiabilidade. No entanto, 0 uso do Image proporcionou linearidade em um intervalo restrito de $163-400 \mathrm{mg} \mathrm{O}_{2} \cdot \mathrm{L}^{-1}$, necessitando de ajustes no modo de aquisição de imagens digitais. Porém, o uso de smartphones para obtenção das imagens permitiu o desenvolvimento de um método muito mais prático, simples e acessível quando comparado ao método padrão referenciado.
\end{abstract}

\section{DETERMINATION OF CHEMICAL DEMAND OF OXYGEN (COD) IN WASTEWATER PER THE ACQUISITION OF DIGITAL IMAGES USING SMARTPHONE}

KEYWORDS: Digital image. Chemical Oxygen Demand (COD). Wastewater.

\begin{abstract}
Chemical Oxygen Demand (COD) is a global indicator of organic matter present in wastewater and surface water, and is widely used in wastewater monitoring the discharge of liquid effluents. The objective of this work was to develop an alternative method for spectrophotometry for COD reading and determination in urban effluents using the acquisition of digital images by smartphone and treatment of them by using ImageJ and Photometrix. Through the construction of analytical curves, it was possible to validate the method using the Photometrix and RGB vector, resulting in a linear working range of $55-600 \mathrm{mg} \mathrm{L}^{-1} \mathrm{O}_{2}$ and a more sensitive, precise and accurate method than the standard method referenced. The use of real samples also obtained better results with this method, with recovery mean of $93.5 \%(n=4)$, able to quantify the analyte in wastewater samples with the same reliability. However, the use of ImageJ provided linearity in a narrow range of $163-400 \mathrm{mg} \mathrm{L}^{-1} \mathrm{O}_{2}$, necessitating adjustments in the acquisition mode of digital images. Although, the use of smartphones to obtain the images allowed the development of a much more practical, simple and accessible method when compared to the standard method referenced.
\end{abstract}

\footnotetext{
${ }_{1}^{1}$ Graduada em Química pela Universidade de Santa Cruz do Sul.

2 Docente do Departamento de Química e Física na Universidade de Santa Cruz do Sul.
} 


\section{INTRODUÇÃO}

No mundo, a disponibilidade dos recursos hídricos está ligada diretamente à qualidade destas águas, visto o fato de que a qualidade da fonte da água delimita os diferentes tipos de usos. Hoje no Brasil, o índice de qualidade da água (IQA), de acordo com a National Sanitation Foundation dos Estados Unidos da América, delimita padrões mínimos de qualidade dos recursos hídricos, onde a poluição destas fontes frente ao despejo de esgoto não tratado são as maiores responsáveis pela degradação da qualidade das águas doces de superfície, uma vez que compostos orgânicos são considerados um dos principais grupos contaminantes ambientais, com danos relativos à saúde humana e aos ecossistemas aquáticos [1].

Além dos parâmetros específicos para controlar a carga poluente em recursos hídricos, os chamados parâmetros gerais são referências da qualidade ambiental, especialmente a demanda química de oxigênio (DQO). Resoluções como a do CONAMA 430/2011[2] e a CONSEMA 355/2017 [3] tem com a DQO um dos parâmetros mais expeditos para determinar a supressão de oxigênio dissolvido das águas residuárias. Responsável por estabelecer o procedimento padrão atual para a determinação da DQO, o Standard Methods for the Examination of Water and Wastewater baseia-se especificamente na utilização do dicromato de potássio como agente oxidante, mas com diferentes formas de procedimento e de detecção[4].

No entanto, devido a matriz complexa e principalmente pela presença de íons cloreto nas amostras de águas residuais, é observada a formação de precipitados na forma de cloreto de prata durante a reação. Deste modo, a formação de precipitados pode causar interferência durante a determinação, visto que o método colorimétrico padrão é extremamente sensível a presença de partículas suspensas [4].

Visto a complexidade dos métodos padrões de determinação da DQO, devido a interferentes e diversas variáveis ao longo das análises, estudos tentam encontrar formas de melhoramento dessa técnica, ou ainda, aprimorando reprodutibilidade, faixa de trabalho, bem como, as reduções de impactos ambientais para execução dos procedimentos analíticos associados. Neste caso, as principais pesquisas direcionam avaliações dos métodos de detecção ou então do método de digestão das amostras [5].

A utilização do ultrassom apresentou resultados excelentes, visto que uma densidade ultrassônica alta em um curto espaço de tempo é capaz de romper as paredes celulares, resultando em alto grau de degradação de compostos e melhorando a sensibilidade das análises de DQO[6], assim como, métodos eletroquímicos para a degradação da matéria orgânica (MO) que aceleram a transferência de massa, expandindo a faixa de trabalho da análise [7] [8]. Métodos com redução nos impactos ambientais também já foram desenvolvidos com boa resposta ao analito, principalmente com a utilização da geração de ozônio e/ou peróxidos como oxidantes e geradores de radicais livres ${ }^{[9][10]}$.

Além dos diferentes métodos para a oxidação da MO, a variação quanto ao modo de detecção destes também são estudados a fim de aumentar a faixa de trabalho, a sensibilidade, exatidão e precisão das análises. 0 óxido de titânio $\left(\mathrm{TiO}_{2}\right)$ e a irradiação de UV também já foram testados como fotocatalisadores, tendo a fluorescência como modo de detecção, através de $\lambda$ de excitação e emissão de 264,8 e362,1 nm [11].

Estudos ainda evidenciam a utilização da quimiluminescência como método de detecção para determinação da DQO, onde um fotomultiplicador detecta a intensidade do sinal das amostras [12]. As grandes indústrias utilizam ainda equipamentos sofisticados e completamente automatizados para o monitoramento dos seus efluentes, abrindo mão de qualquer operação manual necessária. Sistemas esses que são baseados em métodos alternativos, mas com alta sensibilidade e precisão, como sondas fotocatalíticas com UV-Vis e combinada 
com uma fonte ultrassônica para evitar a fixação de sujidades e garantir a confiabilidade dos resultados contínuos [13]

O uso de imagens digitais como ferramenta para análises químicas tem sido amplamente estudado nos últimos anos, utilizando o desenvolvimento da tecnologia como uma ferramenta que facilite o manuseio, transporte, preço e robustez de análises [14]. Para isso é necessário que tal procedimento forneça variação visível de cor no final, onde metodologias que utilizam espectrofotômetro na região do visível, como detecção, são passíveis de estudo para emprego de aquisição de imagens [15].

Protótipo de colorímetro por aquisição de imagens para determinar a concentração de fósforo em águas naturais já foram construídos, reduzindo o tempo de análise e facilitando o transporte do equipamento visto seu tamanho [16]. Assim como a determinação de nitrito em amostras utilizando reagentes de Griess [17] e o monitoramento e caracterização do estado atual do lodo ativado [18].

Os smartphones também já vem sendo utilizados com a finalidade de tornar mais prática e baratas as análises, mantendo a confiabilidade e reprodutibilidade dos resultados. Silva Neto, Fonseca e Braga[19] classificaram as águas minerais de acordo com a concentração e identidade dos íons na amostra empregando a aquisição de imagens digitais por smartphone e tratamento desta com vetor RGB de cor.

A maioria dos métodos que utilizam aquisição de imagens digitais para análises químicas, fazem o uso de softwares computacionais para o tratamento das imagens. Andrade, (2012) ${ }^{[20]}$ estudou a viabilidade do uso de imagens digitais para a determinação de alumínio (III) e cromo (VI) em águas através do software ImageJ, atestando boa concordância entre os valores resultantes do método padrão, com erros relativos baixos.

Apesar de não serem evidenciados estudos referente ao uso de imagens digitais para a determinação da DQO, o método padrão de preparo da amostra apresenta as características e o embasamento teórico necessário para a aplicação dessa técnica, visto que as técnicas de detecção são baseadas na mudança de cor conforme a variação da concentração.

Neste sentido, o presente trabalho buscou desenvolver um procedimento para determinação da DQO, utilizando a aquisição por smartphone e o tratamento de imagens digitais a partir do uso de softwares computacionais e aplicativos móveis. Capaz de reduzir a ação dos interferentes da análise e proporcionando um procedimento confiável, simples e acessível.

\section{MATERIAIS E MÉTODOS}

Os diferentes procedimentos avaliados foram realizados de acordo com o Standart Methods for the Examination of Water and Wastewater, com o método 5220D ${ }^{[4]}$, onde apenas o modo de quantificação foi alterado.

\subsection{Preparo das curvas analítica}

Neste trabalho foram usados tubos de $16 \times 100 \mathrm{~mm}$, e o volume de reagentes de acordo com a tabela 5220:I do Standart Methods [4], onde o volume de reagente está relacionado com o tamanho dos tubos de digestão. A escolha da solução de digestão contendo o dicromato de potássio foi a high range, considerando que as amostras a serem analisadas seriam em concentrações elevadas $\left(\mathrm{mg} \mathrm{L}^{-1} \mathrm{O}_{2}\right)$.

Os tubos fechados contendo cada ponto da curva analítica foram levadas para o digestor, a temperatura de $150^{\circ} \mathrm{C}$ por 2 horas, de modo a se digerir as amostras em refluxo fechado. Após o resfriamento das mesmas, foram realizados os diferentes procedimentos para a quantificação do analito. 
As primeiras curvas analisadas foram retiradas a partir de uma solução estoque padrão de higrogenoftalato de potássio $\left(\mathrm{C}_{8} \mathrm{H}_{5} \mathrm{KO}_{4}\right)$, obtendo diferentes concentrações de $\mathrm{mg} \mathrm{O}_{2} \cdot \mathrm{L}^{-1}$ conhecidas. Posteriormente foram analisadas amostras de efluente bruto da estação de tratamento de efluente da Universidade de Santa Cruz do Sul (UNISC).

\subsection{Método espectrofotométrico}

0 método espectrofotométrico foi baseado na determinação da DQO a partir da absorção de radiação pelos íons $\mathrm{Cr}^{3+}$ em $600 \mathrm{~nm}$, correspondendo ao $\lambda$ máximo.

\subsection{Método por aquisição de imagens}

Dois métodos por aquisição de imagens foram testados, o primeiro utilizando o ImageJ, um software computacional e o segundo um aplicativo móvel de smartphone, o Photometrix. Ambos os métodos utilizam vetores RGB para o tratamento das imagens digitais, que foram adquiridas por smartphone.

\subsubsection{Tratamento das imagens por software computacional}

Para que as imagens digitais fossem geradas, o principal fator a ser controlado foi a influência da luz externa. Assim, foi desenvolvido um protótipo de aparato capaz de neutralizar e impedir a influência da luz externa (Figura 1), que consiste em uma caixa de acrílico preto de $3 \mathrm{~mm}$ de espessura, construída para o encaixe de um tubo de amostra por vez, com dimensões de 150 x 150 × 200 mm. Como fonte de luz, utilizou-se uma spot LED dicroica de $5 \mathrm{~W}$, de luz branca fria, capaz de emitir radiações com $\lambda$ muito próximos ao da luz natural.

Figura 1. Protótipo de aparato para aquisição das imagens digitais. Legenda: 1 - compartimento de fonte de luz; 2 - compartimento de iluminação do tubo; 3 - compartimento de encaixe do tubo; 4 - abertura superior para troca de tubos; 5 - abertura frontal para ajustes; 6 - caminho óptico da luz através do tudo até a saído superior; 7 saída superior para o encaixa da câmera do smartphone e captura da imagem.

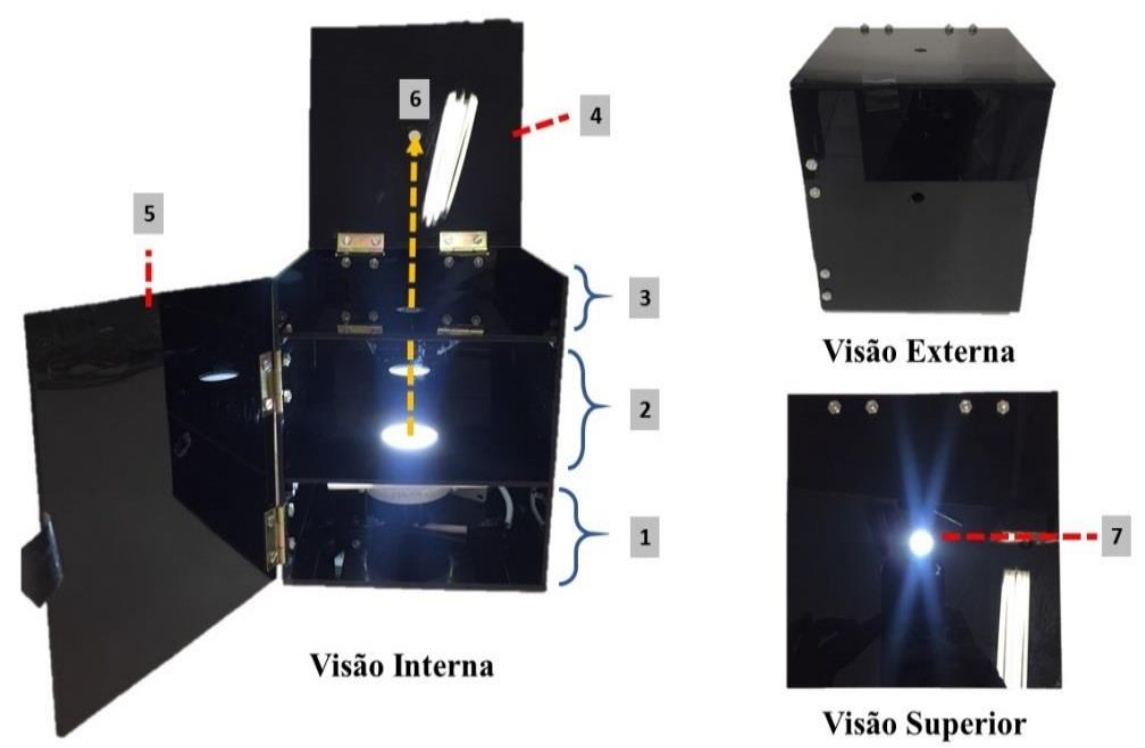

Fonte: Autora. 
As imagens geradas (Figura 2) pelo smartphone foram transferidas para o computador e tratadas, utilizando o software ImageJ - pacote FIJI e pluging para escala macro Readplate [22].

Figura 2. Imagem digital adquirida através do protótipo desenvolvido. Legenda: - área a ser selecionada.

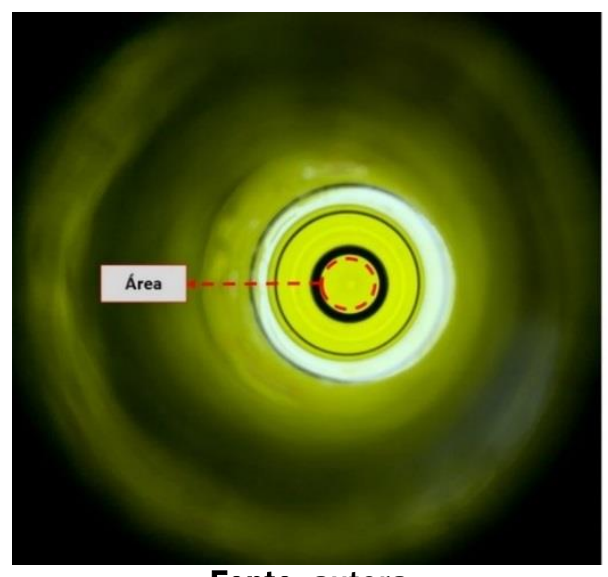

Fonte: autora.

A área central da imagem é selecionada como área a ser tratada, sendo realizado para cada cor do vetor RBG. Como resposta, o software fornece valores de intensidade luminosa e absorbância, além do tamanho da área usada, o desvio padrão, a média, o mínimo e o máximo de valores para intensidade luminosa, onde tanto os valores de absorbância quanto os de intensidade luminosa podem ser usados para quantificar 0 analito.

A partir da resposta de absorbância para cada amostra, foram plotadas 3 curvas distintas para cada cor, vermelha (R), azul (B) e verde (G), afim de identificar qual das cores do vetor possuía correlação direta com a variação da concentração da DQO, ou seja, seletividade para determinar a concentração em amostras reais.

\subsubsection{Tratamento das imagens por aplicativo móvel}

0 modo de aquisição das imagens digitais utilizou apenas um suporte para os tubos, onde o cuidado maior foi em relação a homogeneidade do fundo, que a luz ambiente fosse sempre a mesma e com mesmo ângulo de iluminação, gerando imagens frontais dos tubos (Figura 3).

Figura 3. Imagem adquirida pelo aplicativo móvel.
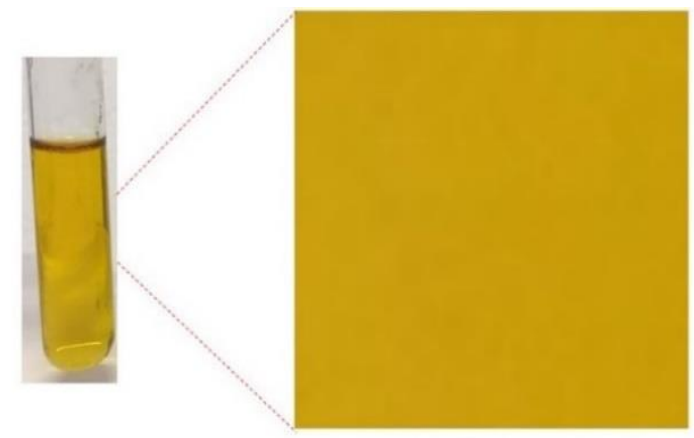

Fonte: autora.

O aplicativo móvel utilizado para a aquisição e tratamento das imagens digitais foi o Photometrix [23]. As curvas analíticas foram plotadas a partir da análise univariada (intensidade x concentração), utilizando como região 
de interesse (ROI) 32 x 32 pixels; resolução de 642 x 480; sem a utilização de flash. Para fins de comparação entre os métodos, a intensidade de luz dada como resposta foi convertida em absorbância (Equação 1) [16].

$$
A=\log \frac{L_{\text {branco }}}{L_{\text {amostra }}}
$$

\subsection{Validação dos métodos}

Para a validação dos diferentes procedimentos, foram avaliados e comparados diferentes atributos em relação ao método padrão colorimétrico (Tabela 1). Como documento orientativo de validação foi utilizado o DoQCGCRE-008, considerando ensaios quantitativos ${ }^{[24]}$.

Tabela 1. Parâmetros necessários para a validação de métodos.

\begin{tabular}{|c|c|c|}
\hline \multirow{2}{*}{ Parâmetros } & \multicolumn{2}{|c|}{ Tipo de ensaio } \\
\hline & Qualitativo & $\overline{\text { Quantitativo }}$ \\
\hline Seletividade & $\bar{X}$ & $\bar{X}$ \\
\hline $\begin{array}{l}\text { Linearidade / faixa de trabalho / Faixa linear de } \\
\text { trabalho / Sensibilidade }\end{array}$ & & $x$ \\
\hline Limite de detecção & $X$ & $x$ \\
\hline Limite de quantificação & & $X$ \\
\hline Tendência / recuperação (Exatidão) & & $X$ \\
\hline Precisão & & $\mathbf{X}$ \\
\hline
\end{tabular}

\section{RESULTADOS E DISCUSSÕES}

\subsection{Aquisição das imagens digitais}

Duas posições para a aquisição das imagens digitais foram testadas, bem como as fontes de luz, até a obtenção de resultados que fornecessem condições necessárias para a confiabilidade do método. A spot LED branca fria de 5W foi escolhida por não demonstrar interferências nas imagens obtidas pelo smartphone e por apresentar espectro de emissão (Figura 4) que abrange os $\lambda$ do visível exigidos para o desenvolvimento do método. A rede de difração de 1000 fendas $/ \mathrm{mm}$ foi utilizada para este teste.

Figura 4 -Espectro de emissão da spot LED branca fria.

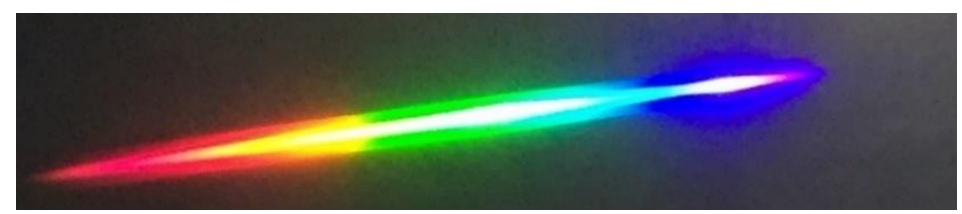

Quanto a posição utilizada para a aquisição das imagens digitais, este foi escolhido por análise visual de homogeneidade das imagens, sendo que as imagens superiores demostraram bons resultados para o método com ImageJ, sem a necessidade de ajustes no protótipo (Figura 5).

Para o método com o Photometrix, o ângulo frontal para a obtenção das imagens foi o utilizado. 
Figura 5 - Imagens digitais adquiridas com ângulo superior por meio do protótipo.
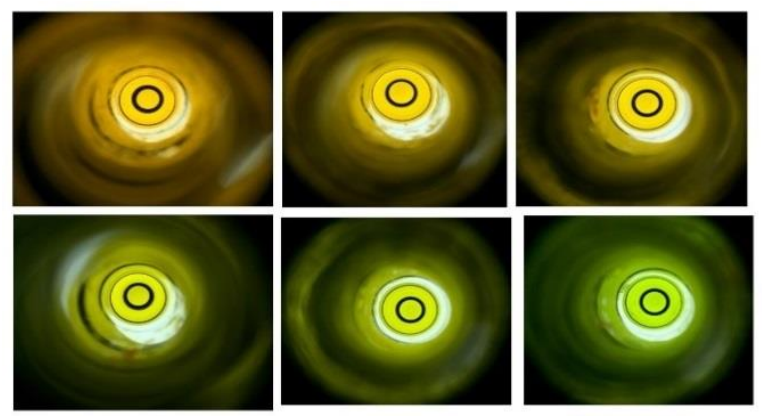

Fonte: Autora.

\subsection{Seletividade}

A comparação das curvas analíticas geradas $(n=7)$ para cada cor do vetor RGB pelo ImageJ (Gráfico 1 ), demostrou que nem todas as cores possuem representatividade para este método.

A partir dos resultados de correlação das curvas, observou-se a seletividade para determinação da DQO quanto ao vetor green $\left(\mathrm{R}^{2}=0,9405\right)$, o qual foi utilizado ao longo das análises.

Gráfico 1. Curvas analíticas para os vetores RGB geradas pelo ImageJ.

Red

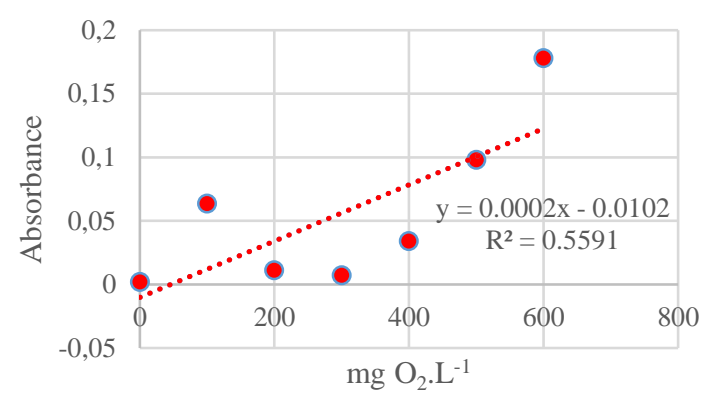

Green

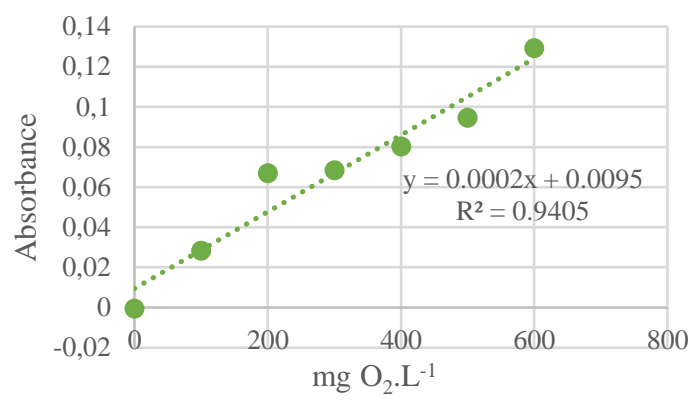

Blue

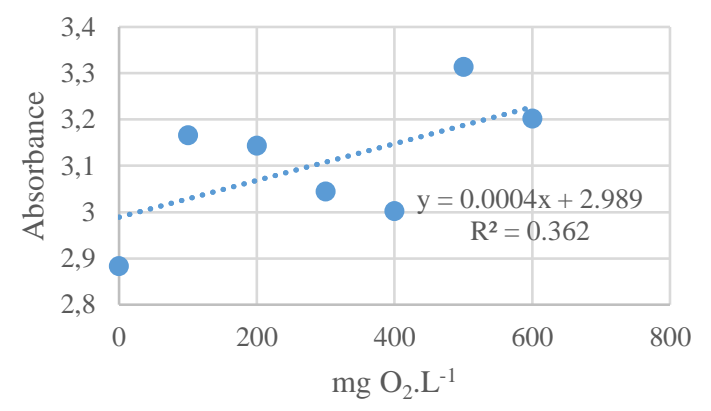

\subsection{Faixa de trabalho e linearidade}

Os dados das curvas analíticas, resultantes de seis curvas analíticas $(n=6)$, demostram linearidade dentro da faixa de trabalho estipulada para ambos os métodos (Gráfico 2). 0 método pelo ImageJ obteve um coeficiente de correlação menor, porém aceitável para a determinação da DQO, sendo que o uso do Photometrix propiciou $\mathrm{R}^{2}$ 
maior, 0,9759 00,00912 (Tabela 2). Valor esse que se aproxima do encontrado em estudo que avaliou a taxa de degradação do $\mathrm{O}_{3}$ para determinar a $\mathrm{DQO}$, obtendo-se $\mathrm{R}^{2}$ de 0,9745 como melhores resultados em um intervalo de trabalho de 0-80 $\mathrm{mg} \mathrm{O}_{2} \cdot \mathrm{L}^{-1}[25]$.

Gráfico 2. Gráficos de linearidade para média de seis curvas padrão.
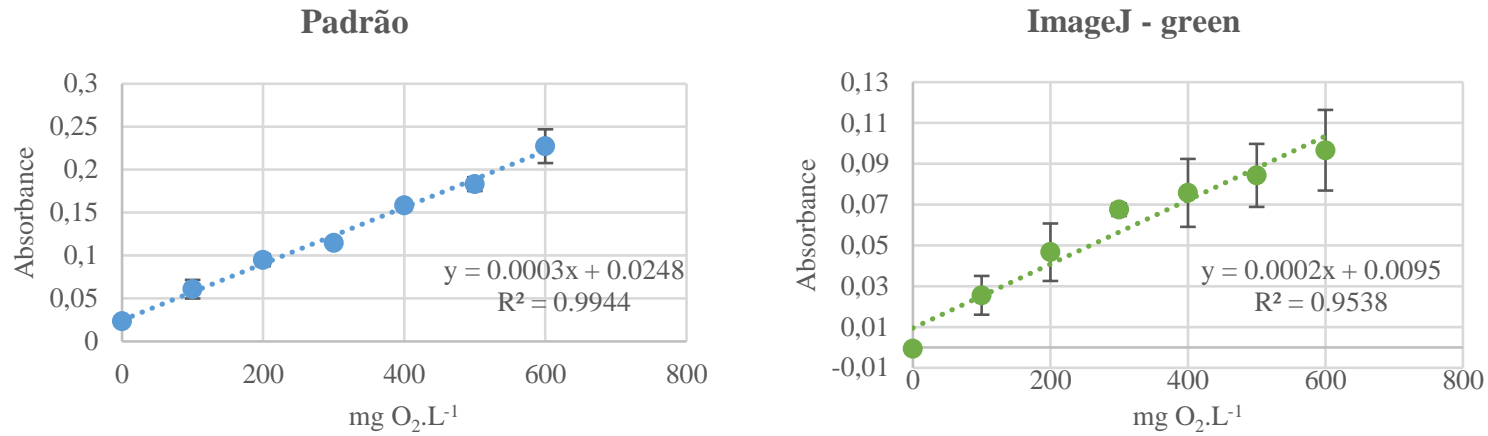

Photometrix

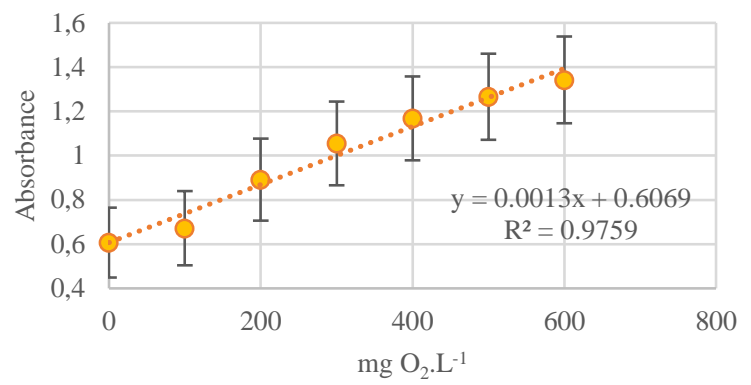

Tabela 2. Curva analítica para o método padrão e o dois desenvolvidos.

\begin{tabular}{|c|c|c|c|c|c|c|}
\hline \multirow[b]{2}{*}{$\mathrm{DQO}\left(\mathrm{mgO}_{2} \cdot \mathrm{L}^{-1}\right)$} & \multicolumn{2}{|c|}{ Padrão } & \multicolumn{2}{|c|}{ ImageJ } & \multicolumn{2}{|c|}{ Photometrix } \\
\hline & Média (abs) & $\pm \mathrm{DP}$ & Média (abs) & $\pm \mathrm{DP}$ & Média (Abs) & $\pm \mathrm{DP}$ \\
\hline 0 & 0,0236 & 0,0004 & $-0,0007$ & 0,0002 & 0,6068 & 0,1579 \\
\hline 100 & 0,0608 & 0,0108 & 0,0255 & 0,0094 & 0,6719 & 0,1677 \\
\hline 200 & 0,0946 & 0,0069 & 0,0466 & 0,0140 & 0,8915 & 0,1854 \\
\hline 300 & 0,1148 & 0,0043 & 0,0675 & 0,0030 & 1,0551 & 0,1893 \\
\hline 400 & 0,1583 & 0,0059 & 0,0757 & 0,0166 & 1,1683 & 0,1895 \\
\hline 500 & 0,1831 & 0,0080 & 0,0843 & 0,0154 & 1,2661 & 0,1945 \\
\hline 600 & 0,2273 & 0,0196 & 0,0966 & 0,0197 & 1,3424 & 0,1961 \\
\hline $\mathrm{n}$ & \multicolumn{2}{|c|}{6} & \multicolumn{2}{|c|}{6} & \multicolumn{2}{|c|}{6} \\
\hline Faixa de trabalho & \multicolumn{2}{|c|}{$0-600$} & \multicolumn{2}{|c|}{$0-600$} & \multicolumn{2}{|c|}{$0-600$} \\
\hline a & \multicolumn{2}{|c|}{0,0003} & \multicolumn{2}{|c|}{0,0002} & \multicolumn{2}{|c|}{0,0013} \\
\hline b & \multicolumn{2}{|c|}{0,0248} & \multicolumn{2}{|c|}{0,0095} & \multicolumn{2}{|c|}{0,6068} \\
\hline R2 & \multicolumn{2}{|c|}{0,9944} & \multicolumn{2}{|c|}{0,9538} & \multicolumn{2}{|c|}{0,9758} \\
\hline
\end{tabular}

Não foi evidenciada variância significativa dos métodos quando comparados ao padrão, uma vez que, os valores de $F$ calculados foram menores que os valores de $F$ crítico para todos os níveis de concentração, considerando nível de confiança de $95 \%$ (Tabela 3). Portanto ambos os métodos possuem faixa de linearidade para concentrações de DQO de 0 a $600 \mathrm{mg} \mathrm{O}_{2} \cdot \mathrm{L}^{-1}$. 
Tabela 3. Análise de variância a partir do teste F.

\begin{tabular}{cccc} 
& & ImageJ & Photometrix \\
\hline Amostras & F crítico & $\mathrm{F}$ & $\mathrm{F}$ \\
\hline $\mathbf{0}$ & 0,198007 & $2,5 \mathrm{E}-09$ & $2,99 \mathrm{E}-08$ \\
100 & 0,198007 & $1,31 \mathrm{E}-06$ & $1,97 \mathrm{E}-05$ \\
200 & 0,198007 & $3,51 \mathrm{E}-06$ & $7,95 \mathrm{E}-06$ \\
300 & 0,198007 & $3,07 \mathrm{E}-07$ & $2,45 \mathrm{E}-06$ \\
400 & 0,198007 & $3,59 \mathrm{E}-07$ & $2,99 \mathrm{E}-06$ \\
500 & 0,198007 & $1,15 \mathrm{E}-06$ & $3,82 \mathrm{E}-06$ \\
600 & 0,198007 & $4,95 \mathrm{E}-06$ & $1,62 \mathrm{E}-05$ \\
\hline
\end{tabular}

\subsection{Sensibilidade}

Perante o valor do coeficiente angular de cada equação da reta (a) (Tabela 2) foi possível comparar a sensibilidade de cada método para a quantificação do analito em questão, onde o método por Photometrix apresentou valor de coeficiente angular maior $(a=0,013)$, constatando a maior sensibilidade em relação ao método padrão e ao ImageJ. Mas de modo geral, os métodos desenvolvidos não apresentaram sensibilidade elevada, onde pequenas variações na concentração do analito também representam pequena variação no sinal medido.

\subsection{Limite de detecção (LD) e quantificação (LQ)}

Para identificar os LD e LQ dos métodos desenvolvidos, considerou-se a médias de respostas para o branco e seus respectivos desvios padrão. Onde o método padrão apresentou LQ de $11,9350_{2} \cdot \mathrm{L}^{-1}$, o método com Photometrix LQ de 55,415 e o método com ImageJ LQ igual a 162,39 (Tabela 4). Deste modo, é possível confirmar que o método com Photometrix é capaz de quantificar concentrações menores do analito, em relação ao ImageJ, sendo o LQ 66\% menor. De modo que foram delimitadas novas faixas lineares de trabalho, considerando o LQ como menor ponto da faixa.

Tabela 4. Limites de detecção e quantificação dos métodos.

Padrão (mg L-1 $\left.\mathrm{O}_{2}\right) \quad$ ImageJ $\left(\mathrm{mg} \mathrm{L}^{-1} \mathrm{O}_{2}\right) \quad$ Photometrix $\left(\mathrm{mg} \mathrm{L}^{-1} \mathrm{O}_{2}\right)$

\begin{tabular}{cccc}
\hline LD & 0,9362 & 19,17 & 17,299 \\
LQ & 11,935 & 162,39 & 55,415 \\
Faixa linear de trabalho & $12-600$ & $163-600$ & $55,5-600$ \\
\hline
\end{tabular}

Pode-se dizer que o método com o ImageJ e o Photometrix apresentam LD e LQ adequado para a quantificação da DQO em efluente, considerando que amostras de efluentes possuem elevada carga de MO, ou seja, altos valores de DQO, o que não exige que os métodos possuam baixos LD e LQ. Sendo que ambos os procedimentos buscam praticidade, enquanto métodos sofisticados, como o uso de sensor térmico que tem objetivo determinar quantidades mais baixa do analito, onde o LD é de $0,74 \mathrm{mg} \mathrm{L}^{-1} \mathrm{O}_{2}{ }^{[26]}$. 


\subsection{Exatidão}

A exatidão dos métodos foi avaliada para três níveis de concentração diferentes, baixa, média e alta, a partir do cálculo de erro relativo (ER) considerando a média dos valores obtidos e o valor admitido como verdadeiro (Tabela 5). Observa-se que o ER para o método padrão se mantem para toda a faixa de concentração, assim como o Photometrix, porém o método desenvolvido proporcionou exatidão maior, entre $7 \%$, metade do encontrado para o método padrão. 0 uso do ImageJ propiciou ER mais baixos nos níveis de concentração menores, sendo que o aumento da concentração acarretou em ER maiores (27\%). Mostrando que o método não é adequado para esses níveis, podendo deixar dúvidas sobre os resultados obtidos.

Tabela 5. Exatidão dos métodos avaliados.

\begin{tabular}{cccc} 
& Padrão & ImageJ & Photometrix \\
\cline { 2 - 4 } $\mathrm{DQO}\left(\mathrm{mg} \mathrm{L}^{-1} \mathrm{O}_{2}\right)$ & \multicolumn{3}{c}{$\mathrm{ER}(\%)$} \\
\hline 200 & 15,27 & 7,04 & 6,58 \\
400 & 13,08 & 17,14 & 6,205 \\
600 & 13,41 & 27,32 & 8,02 \\
\hline
\end{tabular}

Para determinação da DQO, admite-se erro relativo de até $10 \%$, de modo que o método pode ser considerado adequado para a quantificação em determinado nível de concentração.

Portanto, o método desenvolvido utilizando o Photometrix pode ser considerado suficientemente exato para a determinação da DQO na faixa de 55,5-600 $\mathrm{mg} \mathrm{L}^{-1} \mathrm{O}_{2}$ e o método com uso do ImageJ demostrou exatidão em uma faixa mais estreita de $163-400 \mathrm{mg} \mathrm{L}^{-1} \mathrm{O}_{2}$.

Da mesma forma que estudos sobre a avaliação do trajeto óptico de sistemas de absorção de UV consideram baixo o erro medido de $8,7 \%$ pelo método, com $R^{2}$ de 0,9753 em uma faixa de 0-1000 mg L-1 $\mathrm{O}_{2}$ e $\mathrm{LD}^{-1}$ igual a $0,049 \mathrm{mg} \mathrm{0} \cdot \mathrm{L}^{-1}[27]$.

\subsection{Precisão}

A precisão dos métodos foi verificada através da repetitividade entre os mesmos níveis usados para o estudo da exatidão, onde os valores de coeficiente de variação (CV) foram avaliados. A repetitividade dos testes demostraram, em geral, a baixa precisão dos métodos, porém observa-se a tendência de aumento da precisão do método utilizando o Photometrix em concentrações mais elevadas do analito (Tabela 6), indicando a baixa tendência de afastamento das medidas realizadas para o mesmo nível de concentração.

Tabela 6. Repetitividade e precisão dos métodos estudados.

\begin{tabular}{cccc} 
& Padrão & ImageJ & Photometrix \\
\cline { 2 - 4 } $\mathrm{DQO}\left(\mathrm{mg} \mathrm{O}_{2} \cdot \mathrm{L}^{-1}\right)$ & \multicolumn{3}{c}{$\mathrm{CV}(\%)$} \\
\hline 200 & 10,983 & 31,08 & 10,07 \\
400 & 5,59 & 45,17 & 0,82 \\
600 & 11,14 & 22,57 & 2,54 \\
\hline
\end{tabular}




\subsection{Amostras reais}

Foram realizadas quatro amostragens de efluente bruto da estação de tratamento de efluentes (ETE) da Universidade de Santa Cruz do Sul (UNISC), em dias diferentes. Amostras caracterizadas por serem oriundas de águas cinzas e amarelas e apresentarem valores de DQO consideráveis, foram diluídas da amostra em 2 vezes para garantir que a concentração não extrapolasse o último ponto da curva analítica.

As amostras foram tratadas pelos diferentes métodos e avaliadas quanto a recuperação do analito em três diferentes níveis de concentração, com a realização de adição padrão em 100, 400 e 600 mg $0_{2} \cdot L^{-1}$.

A Tabela 7 apresenta os valores de recuperação para as amostras com adição padrão, evidenciando a boa recuperação do analito dentro da própria matriz.

Tabela 7. Teste de recuperação do analito na presença da matriz.

\begin{tabular}{|c|c|c|c|c|c|c|}
\hline & \multicolumn{2}{|c|}{ Padrão } & \multicolumn{2}{|c|}{ ImageJ } & \multicolumn{2}{|c|}{$\begin{array}{c}\text { Photometri } \\
\mathbf{x}\end{array}$} \\
\hline & Média (\%) & DP & Média (\%) & DP & Média (\%) & $\mathrm{DP}$ \\
\hline \multicolumn{7}{|c|}{ Amostras } \\
\hline $25 \%$ & 100 & 0 & 100 & 0 & 100 & 0 \\
\hline $25 \%+100$ & 57 & 7 & 102 & 14,4 & 97 & 2,5 \\
\hline $25 \%+200$ & 80 & 7,7 & 108 & 13,3 & 81 & 2,5 \\
\hline $25 \%+400$ & 81 & 8,4 & 146 & 24,6 & 96 & 4 \\
\hline $\mathrm{n}$ amostral & 4 & & 4 & & 4 & \\
\hline a & 0,0003 & & 0,000 & & 0,0013 & \\
\hline b & 0,0209 & & 0,005 & & 0,54 & \\
\hline $\mathrm{R}^{2}$ & 0,9948 & & 0,944 & & 0,9692 & \\
\hline
\end{tabular}

Através do teste $\mathrm{t}$ (Student), para variância de duas amostras em par para média, comparou-se os mesmos níveis de concentração para amostra em meio a matriz e sem a matriz. Considerando um nível de confiança de 95\% bicaudal, os valores de t para todos os métodos ficaram abaixo do t crítico, evidenciando que o teste t não foi significativo, ou seja, a matriz não causa efeitos sobre o resultado.

\section{CONSIDERAÇÕES FINAIS}

O uso de imagens digitais se mostrou um procedimento aplicável para a determinação da demanda química de oxigênio como um método alternativo para a quantificação do analito. 0 uso de smartphone para a obtenção das imagens, propiciou o desenvolvimento de um método muito mais prático, simples e acessível quando comparado ao método padrão referenciado. Ambos os métodos desenvolvidos, ImageJ e Photometrix, mostramse vantajosos em relação a alguns pontos: não há necessidade de transferência das amostras para uma cubeta, sendo que as imagens são realizadas dos próprios tubos de preparo; redução da interferência nas leituras causada pela precipitação durante a transferência das amostras para a cubeta; redução do volume de resíduos de lavagem gerados, sendo que não há necessidade de lavagem da cubeta; redução do tempo de análise; e baixo custo operacional de instrumentação. 
Os parâmetros avaliados para a validação dos métodos, demostrou que o uso do ImageJ para o tratamento das imagens digitais e quantificação da DQO, necessita de aprimoramento quanto ao modo de aquisição das imagens, porém apresenta boas perspectivas para validação futura. Foi possível validar o método utilizando o aplicativo móvel Photometrix para a determinação da DQO em efluentes, evidenciando ser um método com linearidade de resposta dentro da faixa de trabalho estipulada, com maior sensibilidade, precisão e exatidão comparado ao método espectrofotométrico padrão. Assim ser uma ferramenta alternativa de baixo custo e fácil execução na rotina de controle de qualidade dos efluentes nas ETEs.

\section{AGRADECIMENTOS}

Os autores agradecem ao CNPq pela bolsa IC e o apoio financeiro do Curso de Química da UNISC.

\section{REFERÊNCIAS}

[1] Vieira, L. M. Contaminantes prioritários e emergentes e o saneamento ambiental. 91 f. Trabalho de Conclusão de Curso (Graduação em Engenharia Química) - Universidade Federal de Uberlândia, Uberlândia, 2017.

[2] CONAMA, Resolução. 430/2011. Dispõe sobre condições e padrões de lançamento de efluentes, 2011.

[3] CONSEMA, Resolução 355/2017. Dispõe sobre os critérios e padrões de emissão de efluentes líquidos para as fontes geradoras que lancem seus efluentes em águas superficiais no Estado do Rio Grande do Sul, 2017.

[4] BAIRB, R. B., EATON, A. D., RIOE, E. W. Standard Methods for Examination of Water and Wastewater, 23rd ed. APHA/AWWA/WEF - American Public Health Association; American Water Works Association And Water Environment Federation.ed LCM Pharmabooks, 2017.

[5] BARCELLA, R. A. Estudo comparativo entre diferentes técnicas analíticas para determinação da demanda química de oxigênio (DQO) de rios que recebem efluentes. (2016). http://hdl.handle.net/10183/153268.

[6] LI, X., PENG, Y., HE, Y., JIA, F., WANG, S., \& GUO, S.. Applying low frequency ultrasound on different biological nitrogen activated sludge types: an analysis of particle size reduction, soluble chemical oxygen demand (SCOD) and ammonia release. International Biodeterioration \& Biodegradation (2016), 112, 42-50.

[7] WANG, J., LI, K., YANG, C., WANG, Y., \& JIA, J. Ultrasound electrochemical determination of chemical oxygen demand using boron-doped diamond electrode. Electrochemistry Communications (2012), 18, 51-54.

[8] CHEN, J., LIU, S., QI, X., YAN, S., \& GUO, Q. Study and design on chemical oxygen demand measurement based on ultraviolet absorption. Sensors and Actuators B: Chemical (2018), 254, 778-784.

[9] PISUTPAISAL, N., \& SIRISUKPOCA, U. Development of rapid chemical oxygen demand analysis using ozone as oxidizing agent. Energy Procedia (2014), 50, 711-718.

[10] ESTEVES, L. C., OLIVEIRA, T. R., SOUZA JR, E. C., BOMFETI, C. A., GONÇALVES, A. M., OLIVEIRA, L. C., ... \& RODRIGUES, J. L. A fast and environment-friendly method for determination of chemical oxygen demand by using the heterogeneous Fenton-like process (H202/Fe3- xCoxO4 nanoparticles) as an oxidant. Talanta (2015), 135, 7580.

[11] LI, C., \& SONG, G. Photocatalytic degradation of organic pollutants and detection of chemical oxygen demand by fluorescence methods. Sensors and Actuators B: Chemical (2009), 137(2), 432-436.

[12] YAO, H., WU, B., QU, H., \& CHENG, Y. A high throughput chemiluminescence method for determination of chemical oxygen demand in waters. Analytica chimica acta (2009), 633(1), 76-80.

[13] WTW, T. H. E.; SENSORS, S. Reagent-free measurement directly in the process. (2016), n. 3, p. 8.

[14] SOUZA, F. R., DUARTE-JUNIOR, G. F., GARCIA, P. T., \& COLTRO, W. K. T. Avaliação de dispositivos de captura de imagens digitais para detecção colorimétrica em microzonas impressas. Química Nova, (2014), 37(7), $1171-1176$. 
[15] BENEDETTI, L. P. D. S. Determinação in situ de analitos de interesse alimentício empregando tratamento de imagens digitais de spot tests, (2013).

[16] DA COSTA, A. B., CORREA, G. L. P., ALESSIO, K. O., GARCIA, A. D., ROTHMUND, K., DALBERTO, R., ... \& KIRST, A. Development and Evaluation of a Colorimeter Prototype by Digital Images. REVISTA VIRTUAL DE QUIMICA, (2016), 8(5), 1277-1288.

[17] SHARIATI-RAD, M., IRANDOUST, M., \& MOHAMMADI, S. Multivariate analysis of digital images of a paper sensor by partial least squares for determination of nitrite. Chemometrics and Intelligent Laboratory Systems, (2016), 158, 48-53.

[18] KHAN, M. B., LEE, X. Y., NISAR, H., NG, C. A., YEAP, K. H., \& MALIK, A. S. Digital image processing and analysis for activated sludge wastewater treatment. In Signal and image analysis for biomedical and life sciences (2015), (pp. 227-248). Springer, Cham.

[19] SILVA NETO, G. F., FONSECA, A., \& BRAGA, J. W. Classification of mineral waters based on digital images acquired by smartphones. Química Nova, (2016), 39(7), 876-881.

[20] ANDRADE, S. I. E. D. Um analisador fluxo-batelada baseado em imagem digital para determinação de Al (III) e $\mathrm{Cr}$ (VI) em águas, (2012).

[21] FONSECA, A. P. Iluminação Externa, 1-78. Universidade Federal do Rio de Janeiro.

[22] ImageJ - FIJI, (2018). <https://fiji.sc/>.

[23] HELFER, G. Photometrix, Chemometrics for Android, Windows Phone \& Silverlight, (2018). < http://www.photometrix.com.br>.

[24] DE ACREDITAÇÃO, C. G. DOQ-CGCRE-008-Orientação sobre validação de métodos analíticos, (2011).

[25] PISUTPAISAL, N., \& SIRISUKPOCA, U. Development of rapid chemical oxygen demand analysis using ozone as oxidizing agent, (2014). Energy Procedia, 50, 711-718.

[26] YAO, N., LIU, Z., CHEN, Y., ZHOU, Y., \& XIE, B. A novel thermal sensor for the sensitive measurement of chemical oxygen demand, (2015). Sensors, 15(8), 20501-20510.

[27] CHEN, J., LIU, S., QI, X., YAN, S., \& GUO, Q. Study and design on chemical oxygen demand measurement based on ultraviolet absorption, (2018). Sensors and Actuators B: Chemical, 254, 778-784. 